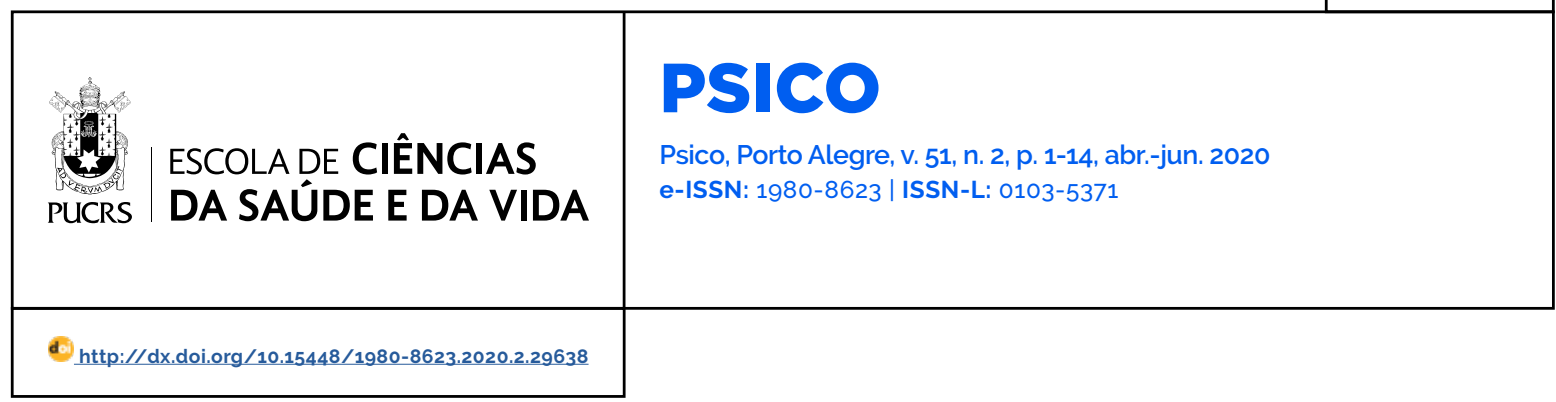

\title{
Estratégias de aprendizagem na educação a distância: revalidação de uma escala
}

\author{
Strategies learning in distance education: escale revalidation \\ Estrategias de aprendizaje en la educación a distancia: revalidación de una escala
}

\section{Elienay Eiko Rodrigues \\ Umekawa $^{1}$ \\ orcid.org/0000-0002-2796-7911 \\ elienay@usp.br}

\section{Thais Zerbini ${ }^{1}$ \\ orcid.org/0000-0001-6799-3658 \\ thais.zerbini@gmail.com}

Recebido em: 10 jan. 2018

Aprovado em: 9 jul. 2019.

Publicado em: 4 set. 2020.

\begin{abstract}
Resumo: Frente a uma nova realidade educacional, o ensino a distância atesta suas contribuições aos processos formativos de modo que compreender o padrão de uso das estratégias de aprendizagem online torna-se fundamental para o aperfeiçoamento do planejamento educacional pois favorece o reconhecimento dos processos individuais de aprendizagem bem como dos procedimentos instrucionais adequados. Este artigo objetiva contribuir com o campo educacional ao verificar evidências de validade da ferramenta "Estratégias de Aprendizagem". Participaram do estudo 126 alunos inscritos em cursos a distância ofertados por instituições de ensino do interior paulista. Após coleta de dados foram efetuadas análises estatísticas de Componentes Principais e Fatoriais, e de consistência interna (alfa de Cronbach). Após validação estatística a escala apresentou quatro fatores: Monitoramento da compreensão, 14 itens ( $\alpha=0,87$ ); Estratégias autorregulatórias, 7 itens $(\alpha=0,80)$; Busca de ajuda interpessoal, 3 itens $(\alpha=0,82)$; Estratégias cognitivo-comportamentais, 7 itens $(\alpha=0,78)$. Os resultados indicam que a escala é estatisticamente válida e confiável.
\end{abstract}

Palavras-chave: educação, ensino a distância, estratégias de aprendizagem.

\begin{abstract}
Understanding the pattern of learning strategies' use becomes fundamental to the improvement of educational planning because it favors the recognition of individual learning processes and the appropriate instructional procedures. This article aims to contribute to the educacional field by verifying validity evidence of the tool "Learning Strategies". Participated in the study 126 students enrolled in distance courses offered by educational institutions in the interior of Sao Paulo. Statistical analyzes of Principal Components and Factors were performed, and internal consistency (Cronbach's alpha). After statistical validation, the scale presented four factors: Monitoring comprehension, 14 items ( $\alpha=0.87$ ); Self-regulatory strategies, 7 items ( $\alpha=$ 0.80); Search for interpersonal help, 3 items $(\alpha=0.82)$; Cognitive-behavioral strategies, 7 items $(\alpha=0.78)$. The results indicate that the scale is statistically valid and reliable. Keywords: education, distance education, strategies of learning.
\end{abstract}

Resumen: Enseñanza a Distancia contribuye a los procesos formativos de modo que comprender el patrón de uso de las estrategias de aprendizaje online se vuelve fundamental para el perfeccionamiento de la planificación educativa pues favorece el reconocimiento de los procesos individuales de aprendizaje bien como de los procedimientos instruccionales adecuados. Este artículo pretende verificar evidencias de validez de la herramienta "Estrategias de Aprendizaje". Participaron del estudio 126 alumnos de cursos a distancia ofrecidos por instituciones del interior paulista. Después de la recolección de datos se efectuaron análisis estadisticos de Componentes Principales y Factoriales, y de consistencia interna (alfa de Cronbach). Después de validación estadística la escala presentó cuatro factores: Monitoreo de la comprensión, 14 items $(\alpha=0,87)$; Estrategias autorregulatorias, 7 items $(\alpha=0,80)$; Búsqueda de ayuda interpersonal, 3 items ( $\alpha$ $=0,82)$; Estrategias cognitivo-conductuales, 7 items $(\alpha=0,78)$. Resultados indican que la escala es estadisticamente válida y confiable.

Palabras clave: educación, enseñanza a distancia, estrategias de aprendizaje. 
Considerando que os cenários contemporâneos de estudo são marcados por alterações diversas, como modificações nos recursos tecnológicos disponiveis, no sistema legal vigente e no processo de veiculação e processamento de informações, constata-se a presença de uma nova lógica de aprendizado e emprego de ferramentas diversificadas (Martins, 2016; Souza, 2018). Cada vez mais os indivíduos são cobrados por competências que envolvem a autoadministração das ações que levam à aquisição do conhecimento; dessa feita, a compreensão acerca dos processos que subjazem à aprendizagem é condição-chave para o êxito nas sociedades atuais (Bjork, Dunlosky, \& Kornell, 2013). Importante elemento nessa nova realidade educativa, a Educação a Distância (EAD) possibilita que um contingente cada vez maior de pessoas não só tenha acesso a conteúdos e informações, mas estabeleça contato, apesar de possiveis distanciamentos físicos (Salas, Tannenbaum, Kraiger, \& Smith-Jentsch, 2012).

Vista como processo de constante construção, a diade ensino-aprendizagem exige do aluno a distância o desempenho de um papel ativo, no qual é fundamental a habilidade de firmar objetivos pessoais e de organizar e controlar os próprios recursos a fim de obter resultados instrucionais satisfatórios. Logo, o elemento autorregulador é imprescindivel para o aprendiz, uma vez que fornece a esse os estratagemas necessários para atingir os resultados almejados (Azevedo et al., 2012).

Atualmente, o aprender a aprender é tido como elemento vital e diferenciador em contextos educacionais, uma vez que o entendimento acerca desse processo favorece a ocorrência de importantes modificações para a aquisição de conhecimento. Nesse sentido, as estratégias de aprendizagem podem ser entendidas como sendo ações sistematizadas utilizadas pelos aprendizes intentando cumprir determinadas tarefas e alcançar metas estabelecidas. Em cenários educacionais que objetivam o alcance do noviciado tais variáveis encontram-se intimamente relacionadas aos processos de aquisição, armazenamento e emprego ulterior dos conteúdos apreendidos.
Oferecendo subsidios ao processamento de novos conhecimentos, as estratégias de aprendizagem se encontram positivamente relacionadas com o estudo exitoso (Alliprandini, Lima, Oliveira, \& Schiavoni, 2012; Beluce \& Oliveira, 2012; Souza, 2010; Zerbini, Abbad, Mourão, \& Martins, 2015). Segundo Costa (2016), tais elementos atuam enquanto fatores influenciadores da atividade educativa, uma vez que ao obter domínio sobre tal processo o aluno passa a contar com habilidades que auxiliarão no cumprimento de objetivos instrucionais.

Indivíduos cônscios tanto dos procedimentos exitosos e malsucedidos, quanto dos passos adotados para empregá-los, podem avaliar, aprimorar e alterar, de forma crítica e decisiva, os próprios processos de ensino (Bjork et al., 2013). A relação positiva existente entre o emprego apropriado de estratégias e um desempenho escolar mais satisfatório é testificada a partir da convergência de resultados de pesquisa advindas de trabalhos como os empreendidos por Oliveira, Boruchovitch e Santos (2011). Considerando que as instituições escolares desempenham uma função essencial no encorajamento ao uso de estratégias apropriadas, é necessário que tais estabelecimentos zelem pela orientação do aprendiz quanto ao emprego e diversificação delas no momento de estudo. Todavia, o entendimento de tais características cognitivocomportamentais ainda se faz limitado (Beluce \& Oliveira, 2012; Martins \& Zerbini, 2014b).

Envolvendo recursos distintos, tais variáveis compreendem uma sucessão de ações variadas sendo possivel, dessa forma, ordenálas de diversos modos. Uma classificação comumente aceita é aquela que diferencia as estratégias de aprendizagem como cognitivas - condutas e pensamentos que permitem que o conhecimento seja armazenado eficazmente - e metacognitivas - procedimentos aplicados pelo sujeito no intuito de planejar, controlar e ajustar seu próprio pensamento. Estando as estratégias de aprendizagem cognitivas voltadas ao cumprimento de tarefas, as estratégias metacognitivas relacionam-se à ordenação, 
regulação e apreciação das primeiras. Esse tipo de monitoramento permite ao estudante verificar se o estratagema utilizado está gerando os resultados pretendidos (Souza, 2010).

Por considerarem que tarefas de natureza e graus de complexidade distintos requerem diferentes estratégias de aprendizagem para ocorrerem, Warr e Allan (1998) formularam um sistema taxionômico constituído por três grandes componentes, os quais compreendem variados niveis de análise, a saber: estratégias cognitivas; estratégias comportamentais; e estratégias autorregulatórias. Vale considerar que a proposta de Warr e Allan (1998), de acordo com Meneses, Abbad, Zerbini e Lacerda (2006), não deve ser compreendida como uma taxionomia, mas como um sistema classificatório de tipos de estratégias diferenciadas, já que os niveis deveriam apresentar necessariamente uma noção de sequência e cumulatividade, ou seja, recairiam em uma classificação e hierarquização.

No tocante à elaboração de estudos voltados à análise do papel desempenhado pelas estratégias de aprendizagem nas mais diversas etapas da escolarização, Boruchovitch et al. (2006) afirmam que embora essa produção venha experimentando crescimento, ainda é considerada insuficiente. Sobretudo no cenário nacional tal escassez se faz notadamente marcada (Costa, 2016).

Entre os instrumentos destinados à aferição de estratégias de aprendizagem encontramse tanto escalas desenvolvidas para o cenário escolar, bem como outras ferramentas pensadas para a formação técnica (Santos, Boruchovitch, Primi, Zenorini, \& Bueno, 2004: Warr \& Downing, 2000). Alguns dos instrumentos utilizados em contextos educacionais se diferenciam pela aplicação exclusiva em etapas específicas da preparação escolar formal, tal como o ensino fundamental (Boruchovitch \& Santos, 2006).

A maior parte das ferramentas construidas corresponde a escalas/questionários que encerram variada gama de estratégias de aprendizagem comportamentais, cognitivas e autorregulatórias. A única exceção diz respeito ao instrumento aberto de autorrelato Self- regulated Learning Interview Schedule (SRLIS); a SRLIS, elaborada por Zimmerman e MartinezPons (1986), foi inicialmente testada em seis diferentes condições de aprendizagem e objetivava avaliar estratégias de aprendizagem autorregulatórias utilizadas por estudantes bem como investigar quais dessas eram mais frequentemente utilizadas, possiveis relações entre elas e desempenho acadêmico.

Em relação à construção de instrumentais a serem empregados em contextos de educação superior, Santos e Boruchovitch (2001) desenvolveram a Escala de Estratégias de Aprendizagem para Universitários (EEA-U). O instrumento é composto por 30 itens relativos às práticas de estudo ou preparação para provas costumeiramente utilizadas por alunos do ensino superior. As questões, dispostas em uma escala do tipo Likert, contam com 4 alternativas de respostas - 4: sempre; 3: às vezes; 2: raramente; 1: nunca - e pontuação variando entre 29 a 116 pontos. Segundo Boruchovitch (2006), após a administração da EEA-U junto a uma amostra de 434 estudantes universitários e realização de análises fatoriais, foram extraídos 5 fatores aptos a explicar $44 \%$ da variância identificada. Em termos de consistência interna, as escalas atingiram niveis satisfatórios nos Fatores $1(\alpha=0.87)$ e $2(\alpha=0.74)$. Todavia, foram encontradas discrepâncias quanto às categorias tradicionais do construto e contradições em relação ao desempenho acadêmico, tomado como critério externo de evidência de validade.

Zerbini, Carvalho e Abbad (2005), baseadas no estudo de Warr e Downing (2000) e guiadas pelas dimensões de Warr e Allan (1998), elaboraram e validaram uma escala de estratégias de aprendizagem. Essa ferramenta, utilizada em pesquisa que objetivou avaliar ações de treinamento a distância, possui 20 itens associados a uma escala do tipo Likert de 11 pontos (o - Nunca a 10 - Sempre). A amostra composta por 1.860 participantes - individuos inscritos em um curso ofertado gratuitamente via web, que pretendia capacitar o aluno a elaborar um plano de negócios - respondeu ao questionário, e os dados obtidos sofreram análises descritivas e exploratórias, bem como análise dos componentes principais e de 
fatoração dos eixos principais. Após a realização das técnicas analíticas, o instrumento apresentou uma estrutura com três fatores: Busca de Ajuda Interpessoal (8 itens, $\alpha=0,85$ ); Elaboração e Aplicação Prática (5 itens, $\alpha=0,75$ ); Repetição, Organização e Ajuda do Material (7 itens, $\alpha=0,78$ ).

Martins (2012), por meio da implementação de seu estudo, validou instrumentos de avaliação de treinamentos a distância, entre eles uma escala de estratégias de aprendizagem (Zerbini \& Abbad, 2008), em contextos de instituições de ensino superior. O questionário foi respondido por 334 participantes através de uma escala de 11 pontos do tipo Likert (o-Nunca a 10-Sempre). As respostas fornecidas foram submetidas à análise dos componentes principais e de fatoração dos eixos principais. $\mathrm{O}$ instrumento apresentou uma estrutura com quatro fatores: estratégias cognitivas (15 itens, $\alpha=0,90$, cargas fatoriais entre 0,33 e 0,72); controle da emoção ( 4 itens, $\alpha=0,77$, cargas fatoriais entre 0,46 e 0,76); estratégias autorregulatórias (7 itens, $\alpha=0,86$, cargas fatoriais entre -0,50 e-0,69); e busca de ajuda interpessoal ( 3 itens, $\alpha=0,68$, cargas fatoriais entre 0,45 e 0,69). A estrutura obtida pela autora diferencia-se empiricamente daquela obtida por Warr e Downing (2000), Zerbini et al. (2005) e Zerbini e Abbad (2008).

Apesar dos avanços envolvendo o estudo das estratégias de aprendizagem em contextos de ensino online, é necessário reconhecer que tais progressos não se fazem suficientes tendo em vista a seriedade do tema à educação (Iglesias \& Salgado, 2012). Faz-se indispensável a constante apreciação dos aspectos que afetam a qualidade e a eficácia das ações educacionais ofertadas. Este estudo intenta contribuir com o campo educacional tendo como objetivo proceder à revalidação estatística da escala denominada "Estratégias de Aprendizagem" (Martins, 2012) em cenários de EAD.

Verifica-se uma limitação envolvendo o empreendimento de trabalhos que contemplem tais aspectos cognitivo-comportamentais no ambiente de ensino. É necessária a consideração dessas variáveis no exercicio de elaboração e de execução de programas educativos, considerando que o emprego de estratégias, por parte dos aprendizes, guarda correspondência com o desempenho escolar, tornando-os mais autorregulados para o enfrentamento das situações de construção do conhecimento (Prates, Lima, \& Ciasca, 2016). Ademais, o entendimento acerca dessa variável também se torna necessário para a efetuação, por parte da equipe pedagógica, de escolhas adequadas quanto a metodologias, meios e procedimentos educacionais tendo em vista o perfil de sua clientela. A fim de que o docente cumpra seu papel formativo é fundamental que haja uma sistematização criteriosa dos objetos de conhecimento, de modo que anterior à transmissão de conteúdos, deve-se primar pelo ensino do aprender a aprender.

\section{Método}

\section{Participantes}

Participaram da presente investigação alunos inscritos em distintas ações educacionais ofertadas por três instituições de ensino superior de caráter público e privado do interior paulista, a saber: Administração, Análise e Desenvolvimento de Sistemas, Ciências Contábeis, Ciências Sociais, Gestão Ambiental, Gestão Financeira, Gestão Pública, Gestão de Recursos Humanos, Logística, Marketing, Processos Gerenciais, Letras, Pedagogia, Sistema de Informação, Teologia, Licenciatura em Ciências e Gestão Estratégica. A população do estudo consistiu no número de inscritos em tais ações instrucionais, totalizando 126 alunos.

No periodo de 29 de novembro de 2012 a 4 de agosto de 2013, foi enviado o questionário de Estratégias de Aprendizagem a todos os participantes. A amostra foi composta predominantemente por individuos do sexo masculino (50,8\%), casados (63,3\%), com filhos $(56,6 \%)$ e residentes na região Sudeste (84,3\%). A idade média encontrada foi de 36,5 anos (DP $=8.53$ ), sendo 32 anos a idade que com mais frequência apareceu, 58 anos a máxima e 19 anos a mínima. No tocante à formação educacional, a maior parcela de participantes contava com o Ensino Superior Completo (61,2\%), 
e majoritariamente, todos os respondentes relataram possuir experiência prévia no uso da internet, denotando domínio das ferramentas utilizadas nos ambientes virtuais.

\section{Características do instrumento}

O instrumento "Estratégias de Aprendizagem" foi revalidado por Martins (2012) e apresentou 31 itens e 4 fatores, com cargas fatoriais variando de -0,69 e 0,76. Os itens do questionário foram associados a uma escala de frequência de comportamento de 11 pontos que varia de o (Nunca) a 10 (Sempre). A seguir, são apresentadas as informações referentes às estruturas empíricas de cada uma dessas escalas obtidas nos estudos correspondentes (Tabela 1).

Tabela 1 - Resumo das informações sobre o instrumento "Estratégias de Aprendizagem".

\begin{tabular}{cccccc}
\hline \multirow{2}{*}{ Instrumento } & Escalas obtidas & No & & \multicolumn{2}{c}{ Cargas fatoriais } \\
\cline { 4 - 6 } & & itens & Alfa & Mín. & Máx. \\
\hline \multirow{2}{*}{$\begin{array}{c}\text { Estratégias de Aprendizagem } \\
\text { (Martins, 2012) }\end{array}$} & Controle da emoção & 4 & 0,77 & 0,46 & 0,76 \\
& Estratégias autorregulatórias & 7 & 0,86 & $-0,5$ & $-0,69$ \\
& Busca de ajuda interpessoal & 3 & 0,68 & 0,45 & 0,69 \\
\hline
\end{tabular}

Fonte: Umekawa (2014).

\section{Coleta de dados}

Respeitados os aspectos éticos envolvidos e mediante aprovação do trabalho pelo Comitê de Ética em Pesquisa da Faculdade de Filosofia, Ciências e Letras de Ribeirão Preto - Universidade de São Paulo (FFCLRP-USP) (protocolo 06615112.1.0000.5407), a coleta de dados foi realizado totalmente a distância, pela internet, por meio da qual os instrumentos uma vez digitalizados foram disponibilizados em formato de questionário para serem administrados via e-mail/ link de pesquisa aos estudantes dos programas de EAD das instituições de ensino superior. As respostas ao questionário foram digitalizadas e integradas ao banco de dados. Uma vez findado o processo de coleta de dados, e obtida uma amostra estatisticamente significativa, as informações foram enviadas para o Statistical Package for the Social Science (SPSS), versão 16.0 - software que viabilizou as análises do material coletado.

\section{Análise de dados}

Foram efetuadas análises descritivas e exploratórias a fim de examinar a exatidão da entrada dos dados, a presença de casos extremos, a distribuição dos casos omissos, a distribuição de frequência e o tamanho das amostras, segundo orientações de Tabachnick e Fidell (2001). Em seguida, deu-se início à validação estatística do instrumento. Para a extração inicial dos fatores foi realizada análise dos componentes principais (principal components - PC). A PC objetiva reduzir o número de variáveis em componentes que expliquem a maior parte da variância original das variáveis. Tal técnica, tida como de simplificação e ortogonalização, analisa os mais importantes componentes e determina o número mínimo de fatores a serem inicialmente extraídos.

Intentando definir o número de componentes, foram empregados na PC três tipos de critérios, a saber: (a) critérios convencionais: apenas foram considerados os valores próprios (eigenvalues) superiores a 1 (um), sendo também analisada a distribuição visual dos mesmos através do scree plot; (b) critérios estatísticos (testes de significância): foi efetuada a análise paralela de Horn, que coteja valores próprios empíricos, resultantes da análise da $\mathrm{PC}$, com valores próprios aleatórios, obtidos por intermédio do software RanEign, em função da quantidade de variáveis 
e do tamanho da amostra. Retira-se da estrutura os fatores que apresentam valor próprio empírico igual ou menor que os valores aleatórios; e (c) critérios de relevância do fator: efetuaram-se análises de interpretabilidade, importância e consistência dos fatores após a rotação dos mesmos. A importância do fator é determinada pela variância que consegue explicar após a rotação da matriz fatorial; de acordo com o critério de Harman considera-se como irrelevante aquele fator que explica menos do que 3\% da variância total das variáveis. O tamanho das cargas fatoriais expressa a validade do fator, ou seja, quanto maiores eles forem, mais a variável é representativa do fator. Todavia, um fator pode ser válido, mas não consistente, por isso, executouse também análises de consistência dos fatores, por meio da análise de Alfa de Cronbach.

\section{Resultados}

As respostas dos 126 participantes aos 31 itens do questionário apresentaram 11 casos extremos univariados e nenhum caso extremo multivariado, os quais foram mantidos no arquivo de dados. Foram identificados valores omissos variando entre 0,7 e 3,9\%. Algumas características foram consideradas: presença de 10 casos para cada variável da ferramenta; existência de relações lineares entre variáveis; e identificação de um par de itens altamente correlacionados entre si, com coeficiente de correlação de 0,81. Para a análise da matriz de covariância em termos de fatorabilidade, foram avaliados o tamanho das correlações e a adequação da amostra. Foram identificados em mais de $50 \%$ dos casos valores de correlação superiores a 0,30, indicando que a matriz provavelmente era fatorizável. No tocante ao teste de Kaiser-Meyer-Olkin (KMO), foi obtido um valor de 0,79, considerado por Pasquali (2004), um bom indice de adequação da amostra.

A análise dos componentes principais, com tratamento listwise para os casos omissos, indicou uma estrutura empírica com 8 componentes que explicaram, em conjunto, 67,90\% da variância total das respostas dos participantes aos itens do questionário. Para tal análise foi seguido o critério dos eigenvalues (valores próprios) maiores ou iguais a 1 (um), bem como o critério de Harman, segundo o qual cada componente deveria explicar, no mínimo, 3\% da variância total. Assim, poderiam ser extraidos oito fatores, no máximo. Em contraposição, pela análise do scree plot (distribuição visual dos valores próprios), foi identificada a presença de três ou quatro componentes, como pode ser observado na Figura 1; já a análise paralela de Horn apontou para uma solução com quatro fatores para o instrumento em questão.

Figura 1 - Distribuição dos valores próprios (scree plot) da escala de Estratégias de Aprendizagem.

\section{Scree Plot}

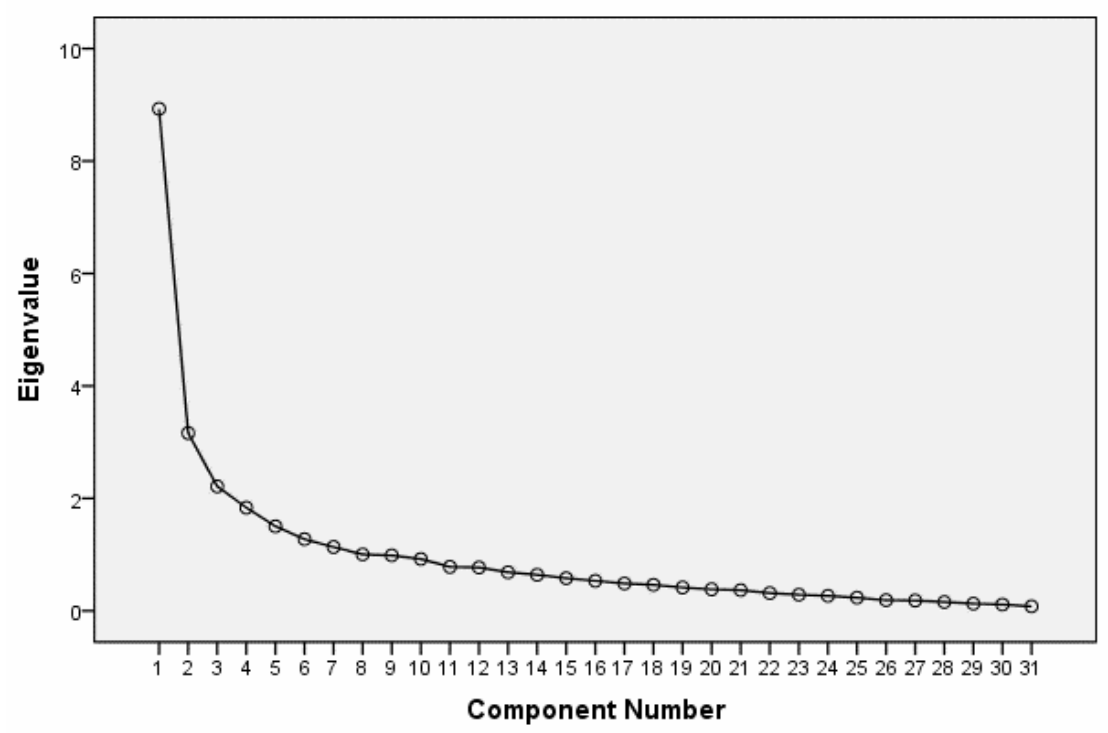


As análises foram realizadas por meio da AF, com método de rotação obliqua e tratamento listwise para casos omissos. Foram incluídos somente os itens com conteúdos semânticos similares e cargas fatoriais superiores ou iguais a 0,30. A partir dessa técnica foram extraídos quatro fatores que explicaram, respectivamente, 28,80\%, 10,19\%, 7,13\% e 5,91\%. A escolha por essa solução apresentou sentido tanto teórico como técnico. Logo, optou-se pela estrutura com quatro fatores, assim como indicado na análise paralela e dos valores próprios. A Tabela 2 apresenta a estrutura empírica da escala, as cargas fatoriais, as comunalidades $\left(\mathrm{h}^{2}\right)$ dos itens, as médias e desvios-padrão, os indices de consistência interna e os valores próprios e percentuais de variância explicada de cada fator.

Tabela 2 - Estrutura empírica da escala de estratégias de aprendizagem (continua).

\begin{tabular}{|c|c|c|c|c|c|c|c|}
\hline \multirow{2}{*}{ Descrição dos Itens } & \multicolumn{4}{|c|}{ Cargas Fatoriais } & \multirow{2}{*}{$h^{2}$} & \multirow{2}{*}{$\mathrm{X}$} & \multirow{2}{*}{ DP } \\
\hline & 1 & 2 & 3 & 4 & & & \\
\hline 1.Mantive a calma quando tive dificuldades. & & 0,71 & & & 0,55 & 7,92 & 2,01 \\
\hline $\begin{array}{l}\text { 2.Repeti a mim mesmo, quando me senti ansioso, que tudo sairia } \\
\text { bem ao final do curso. }\end{array}$ & & 0,55 & & & 0,53 & 7,96 & 2,39 \\
\hline $\begin{array}{l}\text { 3.Mantive a calma com a possibilidade de ter um rendimento } \\
\text { abaixo do esperado. }\end{array}$ & & 0,81 & & & 0,77 & 6,71 & 2,56 \\
\hline $\begin{array}{l}\text { 4.Mantive a calma diante dos erros que cometi ao realizar ativida- } \\
\text { des do curso. }\end{array}$ & & 0,69 & & & 0,75 & 7,11 & 2,42 \\
\hline $\begin{array}{l}\text { 5.Esforcei-me mais quando percebi que estava perdendo a con- } \\
\text { centração. }\end{array}$ & 0,36 & 0,46 & & & 0,70 & 8,08 & 1,84 \\
\hline $\begin{array}{l}\text { 6.Forcei-me a manter a atenção nos estudos quando me senti } \\
\text { desinteressado. }\end{array}$ & 0,36 & 0,47 & & & 0,73 & 7,85 & 2,04 \\
\hline $\begin{array}{l}\text { 7.Esforcei-me mais quando percebi que estava perdendo o inte- } \\
\text { resse no assunto. }\end{array}$ & & 0,35 & 0,43 & & 0,78 & 7,37 & 2,25 \\
\hline $\begin{array}{l}\text { 8.Elaborei perguntas para testar minha compreensão sobre os } \\
\text { conteúdos do curso. }\end{array}$ & 0,47 & & & & 0,60 & 5,8 & 3,02 \\
\hline 9.Revisei a matéria para verificar o quanto eu dominava o conteúdo. & 0,48 & & & & 0,69 & 6,25 & 2,85 \\
\hline $\begin{array}{l}\text { 10.Esforcei-me para verificar minha compreensão sobre o que } \\
\text { estava sendo ensinado. }\end{array}$ & 0,32 & 0,30 & & 0,37 & 0,74 & 7,75 & 2,20 \\
\hline $\begin{array}{l}\text { 11.Busquei auxilio do tutor para esclarecer minhas dúvidas sobre } \\
\text { o conteúdo. }\end{array}$ & & & 0,63 & & 0,55 & 5,27 & 3.05 \\
\hline $\begin{array}{l}\text { 12.Busquei auxilio de colegas nos fóruns para esclarecer minhas } \\
\text { dúvidas. }\end{array}$ & & & 0,72 & & 0,80 & 4,22 & 3,21 \\
\hline $\begin{array}{l}\text { 13.Troquei mensagens com os colegas para esclarecer dúvidas } \\
\text { sobre o conteúdo do curso. }\end{array}$ & & & 0,67 & & 0,71 & 4,6 & 3,48 \\
\hline $\begin{array}{l}\text { 14.Busquei solucionar minhas dúvidas ao consultar as apostilas } \\
\text { do curso. }\end{array}$ & & & & 0,46 & 0,67 & 8,17 & 2,15 \\
\hline $\begin{array}{l}\text { 15.Busquei compreender melhor os conteúdos ao estudá-los nas } \\
\text { apostilas do curso. }\end{array}$ & & 0,37 & & 0,43 & 0,71 & 8,22 & 2,10 \\
\hline 16.Busquei outras fontes de pesquisa, fora da internet, relaciona- & & & & & 0,53 & 7,14 & 3.04 \\
\hline
\end{tabular}




\begin{tabular}{|c|c|c|c|c|c|c|c|}
\hline \multirow{2}{*}{ Descrição dos Itens } & \multicolumn{4}{|c|}{ Cargas Fatoriais } & \multirow{2}{*}{$h^{2}$} & \multirow{2}{*}{$\mathrm{x}$} & \multirow{2}{*}{ DP } \\
\hline & 1 & 2 & 3 & 4 & & & \\
\hline $\begin{array}{l}\text { 17.Busquei sites relacionados ao conteúdo do curso para me } \\
\text { ajudar a aprender. }\end{array}$ & 0,33 & & & & 0.54 & 8,3 & 2,16 \\
\hline $\begin{array}{l}\text { 18.Tentei entender o conteúdo ao aplicá-lo na prática, ao invés de } \\
\text { dedicar tempo lendo ou pedindo ajuda a alguém. }\end{array}$ & 0,64 & & & & 0,47 & 5,89 & 2,87 \\
\hline $\begin{array}{l}\text { 19.Realizei os exercícios práticos propostos ao longo do curso } \\
\text { para me ajudar a aprender. }\end{array}$ & 0,67 & & & & 0,55 & 7,66 & 2,44 \\
\hline $\begin{array}{l}\text { 20.Revisei os conteúdos relativos aos exercícios em que cometi } \\
\text { erros. }\end{array}$ & 0,61 & & & & 0,63 & 6.56 & 2,85 \\
\hline $\begin{array}{l}\text { 21.Aprendi conteúdos ao mentalizá-los repetidamente até perce- } \\
\text { ber que havia entendido. }\end{array}$ & 0,65 & & & & 0,84 & 5,44 & 3,22 \\
\hline $\begin{array}{l}\text { 22. Repeti mentalmente os conteúdos do curso que gostaria de } \\
\text { aprender. }\end{array}$ & 0,70 & & & & 0,83 & 5,77 & 3,26 \\
\hline 23.Fiz anotações sobre o conteúdo do curso. & & & & 0,75 & 0,60 & 7,94 & 2,51 \\
\hline 24.Fiz resumos do conteúdo do curso. & & & & 0,80 & 0,64 & 6.57 & 3,27 \\
\hline 25.Li o conteúdo do curso várias vezes como método para aprender. & & & & 0,41 & 0,56 & 6,01 & 3,02 \\
\hline $\begin{array}{l}\text { 26.Fiz esquemas do conteúdo do curso como método para } \\
\text { aprender. }\end{array}$ & & & & 0,43 & 0,60 & 5.97 & 3,24 \\
\hline
\end{tabular}

Tabela 2 - Estrutura empírica da escala de estratégias de aprendizagem (conclusão).

\begin{tabular}{|c|c|c|c|c|c|c|c|}
\hline \multirow{2}{*}{ Descrição dos Itens } & \multicolumn{4}{|c|}{ Cargas Fatoriais } & \multirow{2}{*}{$h^{2}$} & \multirow{2}{*}{$x$} & \multirow{2}{*}{ DP } \\
\hline & 1 & 2 & 3 & 4 & & & \\
\hline $\begin{array}{l}\text { 27. Refleti sobre as implicações que os conteúdos } \\
\text { aprendidos poderiam ter. }\end{array}$ & 0,47 & & & 0,39 & 0,70 & 7,61 & 2,34 \\
\hline $\begin{array}{l}\text { 28.Busquei desenvolver uma ideia global sobre como } \\
\text { os conteúdos do curso se relacionavam entre si. }\end{array}$ & 0,38 & & & 0,38 & 0,60 & 7,93 & 2,07 \\
\hline $\begin{array}{l}\text { 29.Associei os conteúdos do curso aos meus conheci- } \\
\text { mentos anteriores. }\end{array}$ & & & & 0,38 & 0,49 & 8,69 & 1,67 \\
\hline $\begin{array}{l}\text { 30.Diferenciei, ao analisar os conteúdos do curso, os } \\
\text { aspectos mais importantes dos menos importantes. }\end{array}$ & 0,30 & & & & 0,42 & 7,51 & 2,33 \\
\hline $\begin{array}{l}\text { 31.Identifiquei situações diárias em que eu pudesse } \\
\text { aplicar os conteúdos do curso. }\end{array}$ & & & & & 0,44 & 7,97 & 2,08 \\
\hline N & 105 & 105 & 105 & 105 & & & \\
\hline Eigenvalue (Valor próprio) & 9,38 & 3,07 & 2,17 & 1,84 & & & \\
\hline \% da Variância Explicada & 30,26 & 9,92 & 7,01 & 5.96 & & & \\
\hline No. de itens & 14 & 9 & 4 & 10 & & & \\
\hline $\operatorname{Alfa}(\alpha)$ & 0,87 & 0,80 & 0,82 & 0,78 & & & \\
\hline
\end{tabular}


Para análise da estabilidade, verificou-se o quanto os itens eram bons representantes do fator, por meio do tamanho das cargas fatoriais. Para analisar a interpretabilidade, ao denominar os fatores e associá-los ao agrupamento de itens, recorreu-se ao exame da literatura pertinente. Também foram realizadas análises de consistência interna. A apreciação da consistência dos fatores, por meio da análise do Alfa de Cronbach, seguiu orientações de Pasquali (2004). De um modo geral, o $\alpha$ foi considerando como tendo fiabilidade apropriada e razoável quando o mesmo era da casa de, no mínimo, 0.70. Por último, foram produzidos os escores fatoriais pela média dos escores das variáveis originais que pertencem ao fator.

A escala de Estratégias de Aprendizagem apresentou 31 itens e 4 fatores nomeados: Fator 1 "Monitoramento da Compreensão"; Fator 2 "Estratégias Autorregulatórias"; Fator 3 "Busca de Ajuda Interpessoal"; e Fator 4 "Estratégias Cognitivo-Comportamentais".

O Fator 1, Monitoramento da Compreensão, apresentou um total de 14 itens (5, 6, 8, 9, 10 e 17 a 30, com exceção dos itens 23, 24, 25, 26 e 29), com um bom índice de consistência interna $(\alpha=$ 0,87 ), e cargas fatoriais variando entre 0,30 e 0,70. Dos 14 itens que compuseram esse fator, nove correspondem às estratégias cognitivas e três itens provêm das estratégias autorregulatórias, segundo Martins (2012). No trabalho da referida autora, o fator de estratégias cognitivas é composto por variáveis ligadas à repetição, organização e elaboração do material didático juntamente com um item de aplicação prática e dois itens relativos a estratégias comportamentais de busca de ajuda ao material didático, tal como propõem Warr e Allan (1998). No caso das estratégias autorregulatórias, essas compreendem elementos destinados ao gerenciamento da motivação e monitoramento da compreensão. Nesse estudo, algumas das estratégias cognitivas e autorregulatórias verificadas por Martins (2012) se agruparam em um mesmo fator. Tendo em vista o perfil dos cursos considerados na presente investigação, a amostra contava com diminutos encontros presenciais, ou seja, é bastante coerente o agrupamento de itens que contribuíssem para o processo de aprendizagem e de modificação do comportamento de estudo do discente quando necessário, já que o mesmo respondia majoritariamente pelas ações instrucionais efetuadas.

O Fator 2, Estratégias Autorregulatórias, apresentou um total de sete itens ( 1 a 7) que correspondem à definição de estratégias de controle da emoção e estratégias autorregulatórias, propostas por Martins (2012). De acordo com a definição estabelecida por Warr e Allan (1998), tais itens corresponderiam a estratégias de aprendizagem autorregulatórias no que tange ao controle da emoção - essas estratégias representariam o controle da ansiedade e a prevenção de dispersões de concentração ocasionadas por sentimentos de ansiedade. Novamente, em virtude do feitio da amostra tida em conta, era esperado e necessário que aprendizes, cujas atividades acadêmicas são marcadas pelo distanciamento físico e isolamento social, desenvolvessem recursos hábeis ao domínio emocional e motivacional. Esse fator apresentou um bom indice de consistência interna $(\alpha=0,80)$, com cargas fatoriais variando entre 0,35 e 0,81.

O Fator 3, Busca de Ajuda Interpessoal, contou com um total de três itens (11, 12 e 13) que denotam estratégias voltadas à obtenção de auxílio de outras pessoas, como colegas e tutores, a fim de solucionar dúvidas a respeito do conteúdo do curso. Esse fator retrata um comportamento proativo do aprendiz de procurar por ajuda, ao contrário de obter instruções a partir do próprio material do curso (Warr \& Allan, 1998). Houve correspondência entre o fator em questão e aquele obtido por Martins (2012). O indice de consistência interna para o mesmo é considerado bom $(\alpha=0,82)$, com cargas fatoriais variando entre 0,63 e 0,72.

O Fator 4, Estratégias Cognitivo-Comportamentais, congregou sete itens (14, 15, 23 a 26 e 29), com índice de consistência interna razoável $(\alpha=0,78)$ e itens com cargas fatoriais variando entre 0,38 a 0,80. As estratégias de busca de ajuda ao material didático e repetição, organização e elaboração, na proposta de Warr e Allan (1998), uniram-se em um mesmo fator. A obtenção de informações por meio de fontes que não pressuponham contato social pareceu 
funcionar como recurso auxiliar às estratégias de reflexão ativa acerca dos conteúdos ministrados ao longo das ações instrucionais. Dado que o nível de complexidade de diversos cursos pode ser considerado alto, é razoável ponderar que estratégias responsáveis apenas pela consecução do dado de informação "bruto" não atuem como imprescindiveis à amostra; ao contrário, apesar de fazerem referência aos materiais didáticos empregados, esses são tomados a fim de favorecerem os processos de seleção, decodificação, armazenamento e recuperação de informações.

\section{Discussão}

Tendo em vista os esforços empreendidos por Martins (2012) a fim de elaborar componentes que contemplassem as estratégias de aprendizagem autorregulatórias presentes na escala de Warr e Downing (2000) - que não figuraram em trabalhos como o de Zerbini et al. (2005) - considerouse necessário averiguar como os elementos pertencentes a essa dimensão se comportariam, bem como, aferir a relevância e a contribuição das demais estratégias cognitivas e comportamentais para o desenvolvimento das práticas de estudo dos alunos do nivel superior de ensino. Tal como apregoa Martins (2016) e Zerbini (2007), a atenção dedicada a itens relativos às estratégias de aprendizagem autorregulatórias é justificada com base no pressuposto de que, em cursos a distância via internet, os aprendizes devem demonstrar mais controle emocional, motivacional e um acentuado monitoramento da compreensão para persistir no curso, do que em ações face a face.

Os resultados advindos das análises exploratórias efetuadas não indicaram itens altamente correlacionados entre si. Obtiveram-se quatro fatores de estratégias de aprendizagem os quais apresentaram maiores similaridades com as dimensões teóricas propostas por Warr e Allan (1998), do que com as dimensões e estruturas obtidas nos estudos de Martins (2012), Zerbini et al. (2005) e Zerbini e Abbad (2008).

No fator Monitoramento da compreensão (14 itens, $\alpha=0,87$, cargas fatoriais entre 0,30 a 0,70 ) as estratégias de aquisição de aprendizagem propostas por Warr e Allan (1998) foram agregadas em um único fator. Tal estrutura difere da obtida por Martins (2012), cujas estratégias de monitoramento da compreensão associaram-se a itens de controle da motivação e de busca de ajuda ao material didático - compondo o fator denominado Estratégias autorregulatórias, 7 itens, $\alpha=0,86 \mathrm{e}$ cargas fatoriais entre -0,50 e-0,69. A extração de um fator unicamente composto por estratégias voltadas ao monitoramento da compreensão revela que frente às singularidades dos cursos a distância (encontros presenciais esparsos delegando ao aluno a inspeção primária dos processos de estudo), os participantes devem não somente recorrer, mas desenvolver competências hábeis de gerenciamento que lhes permitam automonitorar e autoavaliar seus processos de aprendizagem. Especialmente em cenários educacionais a distância, recursos favorecedores do estudo autônomo do aprendiz estão sendo cada vez mais retratados como cruciais à "sobrevivência" do estudante em situações nas quais ele é o principal responsável pelo exame minucioso e sistemático das atividades que alicerçam sua progressão no evento instrucional não presencial (Gureckis \& Markant, 2012; Martins \& Zerbini, 2014a; Mello, 2018; Santoro \& Bido, 2017). Tal como defendido por Carrasco e Silva (2017), a capacidade de aprender passou a ser um relevante item enquanto o tempo de aprendizagem tornouse constante e o pensamento, questionamento, imaginação e criatividade são, atualmente, essenciais (Martins, 2016).

O segundo fator Estratégias autorregulatórias (7 itens, $\alpha=0,80$, cargas fatoriais entre 0,35 a 0,81 ) guarda correspondências com os fatores obtidos por Martins (2012), Warr e Downing (2000) e Zerbini e Abbad (2008). Tais estratégias vinculam-se ao controle da ansiedade, atenção e motivação. Nesse estudo, o fator foi formado por itens derivados das estratégias Controle da emoção e Controle da motivação presentes na clássica definição de Warr e Allan (1998). Considerando que o êxito estudantil não depende unicamente de atributos acadêmicos, mas também do emprego de adequados recursos controladores 
dos estados emocionais do sujeito que aprende face a dificuldades, elementos estressantes ou interesses concorrentes, é possivel pensar que para a amostra considerada foi fundamental a utilização de tais variáveis, que auxiliou na resposta a desafios de seleção, focalização e processamento de estímulos.

O fator Busca de ajuda interpessoal ( 3 itens, $\alpha$ = 0,82 , cargas fatoriais entre 0,63 a 0,72) congrega estratégias que exprimem a busca ativa por auxílio de outros indivíduos, como pares e tutores, para sanar dúvidas sobre o conteúdo do curso (Warr \& Allan, 1998). Esse fator se fez presente nos estudos conduzidos por Martins (2012), Warr e Downing (2000) e Zerbini e Abbad (2008) - cujas propriedades foram, respectivamente: três itens, $\alpha=0,68$, cargas fatoriais entre 0,45 a 0,69; cinco itens, $\alpha=0,84$, cargas fatoriais entre 0,54 a 0,68; seis itens, $\alpha=0,89$, cargas fatoriais entre 0,66 a 0,83 .

O quarto fator Estratégias cognitivocomportamentais (sete itens, $\alpha=0,78$, cargas fatoriais entre 0,38 a 0,80 ) agrupou itens pertencentes a duas estratégias primárias, a saber: repetição, elaboração e organização e busca de ajuda ao material didático. De acordo com Warr e Allan (1998), as estratégias cognitivas de repetição consistem na repetição mental da informação na forma em que foi apresentada ao aluno; as estratégias de organização se referem à identificação de ideias centrais do material e à criação de esquemas mentais que agrupam e relacionam elementos que foram aprendidos; e as estratégias de elaboração correspondem aos procedimentos de análise e de reflexão acerca de implicações e conexões existentes entre o material aprendido e o arcabouço de informações já existentes. Já a estratégia de busca de ajuda ao material didático implica a obtenção de orientações a partir de consultas a fontes que não envolvam contato social. O arranjo desse fator diferiu do encontrado por Martins (2012), Warr e Downing (2000) e Zerbini e Abbad (2008).

Observando as definições conceituais atribuidas às estratégias que compõem o último fator extraído, essas se revelaram coadunadas às dimensões teóricas propostas por Warr e Allan (1998). Além de se originarem a partir de um radical comum (o grande grupo das estratégias de aprendizagem primárias), o agrupamento encontrado indicou que a busca de informações por meio de materiais educativos parece atuar como forma de aprimorar os processos de reflexão ativa dos objetos de aprendizagem vistos. Ademais, considerando as propriedades da amostra deste estudo, torna-se possivel que as estratégias relativas a consultas ao material didático tenham sido compreendidas como demasiadamente simples para ações educativas que exibem objetivos educacionais de niveis mais complexos. Dessa feita, tais estratégias não ensejaram o surgimento de um fator distinto para as mesmas.

A expressiva oferta de eventos educativos a distância sinaliza para a necessidade de discutir sua efetividade e qualidade através de reflexões científicas, sendo o padrão de uso de estratégias de aprendizagem dos estudantes um dos elementos cruciais a serem investigados. Uma vez que maximizam a aquisição e o armazenamento de novos conhecimentos, tais elementos repercutem significativamente nas ações educacionais (Costa, 2016; Martins, 2016; Zerbini, Abbad, Mourão, \& Martins, 2015). Nesse sentido, a execução de atividades interativas e complementares, como aquelas ocorridas em processos de ensino, exige de ambos os personagens - aluno e docente - o entendimento, o domínio e o uso de estratégias de aprendizagem; se por um lado, o emprego eficaz desses recursos favorece a competência do aprendiz para o enfrentamento de situações de estudo, da perspectiva do professorado tal conhecimento robustece a formação inicial dos mesmos (Beluce \& Oliveira, 2016).

Para que tais informações sejam levantadas se faz necessário a construção, testagem e uso de instrumentos de medida específicos à EAD. Dessa forma, os resultados encontrados fornecem contribuições para investigações acerca do planejamento instrucional, visto que sinalizam quais são as estratégias de aprendizagem mais empregadas pelos estudantes para executar tarefas escolares quando mediadas pelas novas tecnologias da informação e comunicação. Entre as limitações do estudo, destacam-se 
o número reduzido da amostra, bem como a necessidade de que a ferramenta seja reaplicada em outros contextos. Apesar das variações entre as médias dos escores dos diferentes itens da escala indicarem uma predominância no uso de determinadas estratégias de aprendizagem, recomenda-se a efetuação de análises específicas de comparação de médias, como por exemplo GLM de medidas repetidas.

Apesar das limitações, são oferecidos subsidios para o aprimoramento do entendimento acerca das estratégias de aprendizagem. O entendimento a respeito dos padrões de uso e de preferências das estratégias de aprendizagem possibilita cotejar o ajustamento e a conformidade das estratégias de ensino promovidas pelas instituições de ensino através de atividades e de conduções pedagógicas àquelas realmente adotadas pelos alunos, contribuindo, dessa feita, para que haja consonância entre ambas. Em sintese, para ambientes de ensino online, é necessária uma compreensão mais acurada acerca das estratégias de aprendizagem tendo em vista que essas concorrem para a interlocução entre docentes, alunos e objetos de conhecimento; para o robustecimento da percepção de vínculo acadêmico; e, por fim, para o desenvolvimento de habilidades voltadas ao monitoramento da aprendizagem, ao gerenciamento do tempo de estudo e à autonomia.

\section{Referências}

Alliprandini, P. M. Z., Lima, C. F., Oliveira, D. E. B., \& Schiavoni, A. (2012). Diferenças entre gênero no uso de estratégias de aprendizagem na educação a distância. Trabalho apresentado no IXANPED SUL. Caxias do Sul. Anais do IX Anped Sul, p. 1-14.

Azevedo, A. S., Dias, P. C., Salgado, A., Guimarães, T., Lima, I., \& Barbosa, A. (2012). Relacionamento Professor-Aluno e Auto-Regulação da Aprendizagem no $3^{\circ}$ Ciclo do Ensino Médio Português. Paidéia, 22(52), 197-206. https://doi.org/10.15.90/S0103-863X2012000200006.

Beluce, A. C., \& Oliveira, K. L. (2016). Escala de estratégias e motivação para aprendizagem em ambientes virtuais. Revista Brasileira de Educação, 21(66), 593-610. https:// doi.org/10.1590/S1413-24782016216631.

Beluce, A. C., \& Oliveira, K. L. (2012). Ambientes virtuais de aprendizagem: das estratégias de ensino às estratégias de aprendizagem. Trabalho apresentado no IX ANPED SUL. Caxias do Sul. Anais do IX Anped Sul, p. 1-14.
Bjork, R. A., Dunlosky, J., \& Kornell, N. (2013). Self-Regulated Learning: Beliefs, Techniques, and Illusions. Annu. Rev. Psychol., 64, 417- 444. https://doi.org/10.1146/ annurev-psych-113011-143823.

Boruchovitch, E. (2014). Autorregulação da aprendizagem: contribuições da psicologia educacional para a formação de professores. Psicol. Esc. Educ., 18(3), 401409. https://doi.org/10.1590/2175-3539/2014/0183759.

Boruchovitch, E. (2006). Avaliação psicoeducacional: desenvolvimento de instrumentos à luz da Psicologia Cognitiva baseada na teoria do processamento da informação. Avaliação Psicológica, 5(2), 145-152. (DOI INEXISTENTE)

Boruchovitch, E., \& Santos, A. A. A. (2006). Estratégias de aprendizagem: conceituação e avaliação. In A. P. P. Noronha, \& F. F. Sisto (Org), Facetas do fazer em avaliação psicológica (pp. 107-124). São Paulo: Vetor.

Brandão, H. P., \& Borges-Andrade, J. E. (2011). Desenvolvimento e Validação de uma Escala de Estratégias de Aprendizagem no Trabalho. Psicologia: Reflexão e Critica, 24(3), 448- 457. https://doi.org/10.1590/S010279722011000300005 .

Carrasco, T. S., \& Silva, F. M. (2017). Informal learning at work context: a meta-study of brazilian scientific production. Revista de Administração Mackenzie-RAM (Mackenzie Management Review), 18(4), 137-163. https://doi.org./10.1590/1678-69712017/administracao. v18n4p137-163 https://doi.org/10.1590/1678-69712017/ administracao.v18n4p137-163.

Costa, E. R. (2016). As estratégias de aprendizagem como forma de promover a interdisciplinaridade e o aprender a aprender: investigando as estratégias de aprendizagem de universitários dos cursos de história e Psicologia. Revista EdaPECl, 16(3), 486-500. (DOI INEXISTENTE)

Costa, E. R., Boruchovitch, E. (2009). As estratégias de aprendizagem e a produção de textos narrativos. Psicologia: Reflexão e Critica, 22(2), 173-180. https://doi. org/10.1590/S0102-79722009000200002.

Gureckis, T. M., \& Markant, D. B. (2012). Self-Directed Learning: A Cognitive and Computational Perspective. Perspectives on Psychological Science, 7(5), 464-481, 2012. https://doi.org/10.1177/1745691612454304.

Iglesias, M., Salgado, J. F. (2012). Effectiveness of Occupational Training Through Videoconferencing: Comparison with Classroom Training and Individual Differences. Revista de Psicología del Trabajo y de las Organizaciones, 28(3), 183-188. https://doi.org/10.5093/tr2012a15.

Joly M. C. R. A., Silva, D. V., Ferreira-Rodrigues, C. F., Bueno, J. M. P., \& Almeida, L. S. (2015). Competência de estudo para uma amostra universitária da área de exatas. Revista Quadrimestral da Associação Brasileira de Psicologia Escolar e Educacional, 19(1), 23-29. https:// doi.org/10.1590/2175-3539/2015/0191790.

Martins, L. B. (2012). Aprendizagem em ações educacionais a distância: fatores influentes no desempenho acadêmico de universitário. (Dissertação de mestrado). Faculdade de Filosofia, Ciências e Letras de Ribeirão Preto, Universidade de São Paulo). 
Martins, L. B. (2016). Impacto do treinamento no trabalho: determinantes individuais e contextuais em cursos corporativos a distância. 2016. (Tese de Doutorado). Faculdade de Filosofia, Ciências e Letras de Ribeirão Preto, Universidade de São Paulo, São Paulo.

Martins, L. B., \& Zerbini, T. (2014a). Educação a distância em instituições de ensino superior: uma revisão das pesquisas. Revista Psicologia: Organizações e Trabalho, 14(3), 271-282. (DOI INEXISTENTE)

Martins, L. B., \& Zerbini, T. (2014b). Escala de Estratégias de Aprendizagem: evidências de validade em contexto universitário híbrido. Psico-USF, 19(2), 317-328. https:// doi.org/10.1590/1413-82712014019002007.

Mello, C. G. (2017). Estratégias de aprendizagem em ações educacionais à distância: relação com caracteristicas da clientela e reações ao curso. (Dissertação de Mestrado). Faculdade de Filosofia, Ciências e Letras de Ribeirão Preto, Universidade de São Paulo, São Paulo.

Meneses, P. P. M., Abbad, G., Zerbini, T., \& Lacerda, E. (2006). Medidas de características da clientela em avaliação de TD\&E. In J. E. Borges-Andrade, G. Abbad, \& L. Mourão (Org.), Treinamento, desenvolvimento e educação em organizações e trabalho: fundamentos para a gestão de pessoas (pp. 422-442). Porto Alegre: Artmed.

Oliveira, K. L., Boruchovitch, E., \& Santos, A. A. A. (2011). Estratégias de aprendizagem no ensino fundamental: análise por gênero, série escolar e idade. Psico, 42(1), 98-105. (DOI INEXISTENTE)

Pasquali, L. (2004). Análise fatorial para pesquisadores. Petrópolis: Vozes.

Prates, K. C. R., Lima, R. F., \& Ciasca, S. M. (2016). Estratégias de aprendizagem e sua relação com o desempenho escolar em crianças do Ensino Fundamental I. Revista DE psicopedagogia, 33(100), 19-27. (DOI INEXISTENTE)

Santoro, S. \& Bido, D. S. (2017). Estratégias de aprendizagem informal no trabalho utilizadas por gerentes em uma Instituição Financeira Pública. Rev. Elet. Gestão e Serviços, 8(1), 1878-1901. https://doi.org/10.15603/21777284/regs.v8n1p1878-1901.

Salas, E., Tannenbaum, S. I., Kraiger, K., \& Smith-Jentsch, K. (2012). The Science of Training and Development in Organizations: What Matters in Practice. Psychological Science in the Public Interest, 13(2), 74-101. https://doi. org/10.1177/1529100612436661.

Santos, A. A. A., \& Boruchovitch, E. (2001). Escala de avaliação de estratégias de aprendizagem em universitários. Universidade São Francisco: Bragança Paulista.

Santos, A. A. A., Boruchovitch, E., Primi, R., Zenorini, R. P. C., \& Bueno, J. M. H. (2004). Escala de avaliação de estratégias de aprendizagem para universitários (EAP-U): aplicação do Modelo de Rasch de créditos parciais. Psicologia: Teoria, Investigação e Prática, 9(2), 227-242. (DOI INEXISTENTE)
Sousa, A. A. D. (2018). Preditores da aprendizagem e instrumentos de transferência de treinamento: um estudo com profissionais da saúde pública. (Tese de Doutorado). Faculdade de Filosofia, Ciências e Letras de Ribeirão Preto, Universidade de São Paulo, São Paulo.

Souza, L. F. N. I. (2010). Estratégias de aprendizagem e fatores motivacionais relacionados. Educar, 36, 95-107. https://doi.org/10.1590/S0104-40602010000100008.

Tabachinick, B.G., \& Fidell, L.S. (2001). Using multivariate statistics. New York: Harper-Collins College Publishers.

Warr, P., \& Allan, C. (1998). Learning strategies and occupational training. Internacional Review of Industrial and Organizational Psychology, 13, 83-121. (DOI INEXISTENTE)

Zerbini, T. (2007). Avaliação da transferência de treinamento em curso a distância. (Tese de doutorado). Instituto de Psicologia, Universidade de Brasilia.

Zerbini, T., \& Abbad, G. (2008). Estratégias de aprendizagem em curso a distância: validação de uma escala. Psico-USF, 13, 177-187. https://doi.org/10.1590/S141382712008000200005 .

Zerbini, T., Abbad, G., Mourão, L., Martins, L. B. (2015). Estratégias de Aprendizagem em Curso Corporativo a Distância: como Estudamos Trabalhadores?. Psicologia, Ciência e Profissão, 35(4), 1024-1041. https://doi. org/10.1590/1982-3703000312014.

Zerbini, T., Carvalho, R. S., Abbad, G. (2005). Treinamento a distância via internet: construção e validação de escala de estratégias de aprendizagem. Trabalho apresentado no XXIX ENANPAD Associação Nacional dos Programas de Pós-Graduação em Administração, Brasilia.

Zerbini, T., Abbad, G., Mourão, L., \& Martins, L. B. (2015). Estratégias de Aprendizagem em curso corporativo a distância: como estudam os trabalhadores?. Psicologia: Ciência e Profissão, 35(4), 1024-1041. https://doi. org./10.1590/1982-3703000312014.

Zimmerman, B. J., \& Martinez-Pons, M. (1986). Development of a structured interview for assessing student use of self-regulated learning strategies. American Educational Research Journal, 23, 614-628. https://doi. org/10.3102/00028312023004614

\section{Elienay Eiko Rodrigues Umekawa}

Mestre em Psicologia pela Faculdade de Filosofia, Ciências e Letras de Ribeirão Preto da Universidade de São Paulo (USP, Ribeirão Preto, SP, Brasil), analista com formação em Psicologia do Instituto Nacional do Seguro Social (INSS) em Ribeirão Preto, SP, Brasil.

\section{Thaís Zerbini}

Doutora em Psicologia pela Universidade de Brasília (UnB, Brasilia, DF, Brasil), docente - professora associada da Universidade de São Paulo (USP) em Ribeirão Preto, SP, Brasil 


\section{Endereço para correspondência}

Elienay Eiko Rodrigues Umekawa

Rua Drº Loiola, 864

Vila Tibério

14050-070

Ribeirão Preto, SP, Brasil 